\title{
Qualitative and quantitative evaluation of the ground movement risk on an urban cartographic scale Example of QUEMADO (Eastern Rif, Morocco)
}

\section{Evaluation qualitative et quantitative du risque 'mouvement de terrain' à une échelle cartographique urbaine Cas du versant QUEMADO (Rif oriental du Maroc)}

\author{
RFIFI Mohamed ${ }^{1, a}$, AIT BRAHIM Lahsen ${ }^{1}$
}

${ }^{1}$ Université Mohammed V, Faculté des sciences, Unité de recherche GEORISK, LGRN, 4 Avenue Ibn Battouta -Agdal, BP 1014, Rabat, Maroc.

\begin{abstract}
The morphostructural examination of QUEMADO slope, contributes to distinguish the vulnerability indications that refer to the ground movements. They are supported by the combination of geological, geo morphological, hydro geological parameters. In addition, the seismic activity that is known in this area increases the vulnerability of the coastal areas. Thus, many cracks and locatable indices in the studied area constitute a witnesses of movement risk that threat directly the projects, the installations and the urban constructions of Al Hoceima city, in proximity of the studied cliff.

The present study is consecrated to achieve a comparative study of the evaluated risk "ground movement" by two methodological approaches. The qualitative approach which is most usually employed, and the quantitative approach that requires a scientific effort of adaptation and reproducibility. The risk evaluation is based on using four data components; mainly the topographical slope, the lithology, the geomorphology and the ground occupation. The results were interpreted by a discrete analysis of the unstable areas through the land reconnaissance

Keywords: Morocco, Western Rif, QUEMADO, Ground movements, Vulnerability, Evaluation, Methodological approach.
\end{abstract}

\section{Introduction}

Les mouvements de terrain au Rif ont fait l'objet de plusieurs travaux, dont ceux de [1]. D'autres travaux consacrés à des régions précises du Rif, ont invoqué la combinaison la combinaison de plusieurs facteurs provoquant l'instabilité de terrain [2, 3, 4, 5]. Cependant des études sur les mouvements dans les pentes signalent que le facteur sismique peut être à l'origine des surpressions interstitielles [6].

L'objet de la présente étude est de mettre en évidence la conjonction entre divers facteurs déclencheurs d'instabilité à l'échelle de la zone de QUEMADO, l'évaluation de sa vulnérabilité au risque de mouvement de terrain par l'emploi de deux approches d'étude qualitative et quantitative ayant fait l'objet d'études antérieures [7, 8, 9], cette analyse fera l'objet également d'une reconnaissance terrain.

\section{Terrain d'étude}

\subsection{Cadre géologique}

La région d'Al Hoceima appartient à la zone interne des unités structurales du Rif résultantes de l'orogénèse de la chaine alpine. Le Rif interne se compose d'un ensemble d'unités allochtones alpines, empilées les unes sur les autres et séparées par des contacts anormaux [10]. Parmi ces unités on trouve la dorsale calcaire externe qui supporte, sous forme de klippe tectonique, les terrains de la dorsale calcaire interne et ceux de la nappe paléozoïque Ghomarides. Ces unités reposent sur les terrains de la dorsale prédorsalienne (semelle tertiaire). L'ensemble de ces formations chevauchent vers le sud les flyschs de Tisirène $[11,12,13]$ (figure 1). 


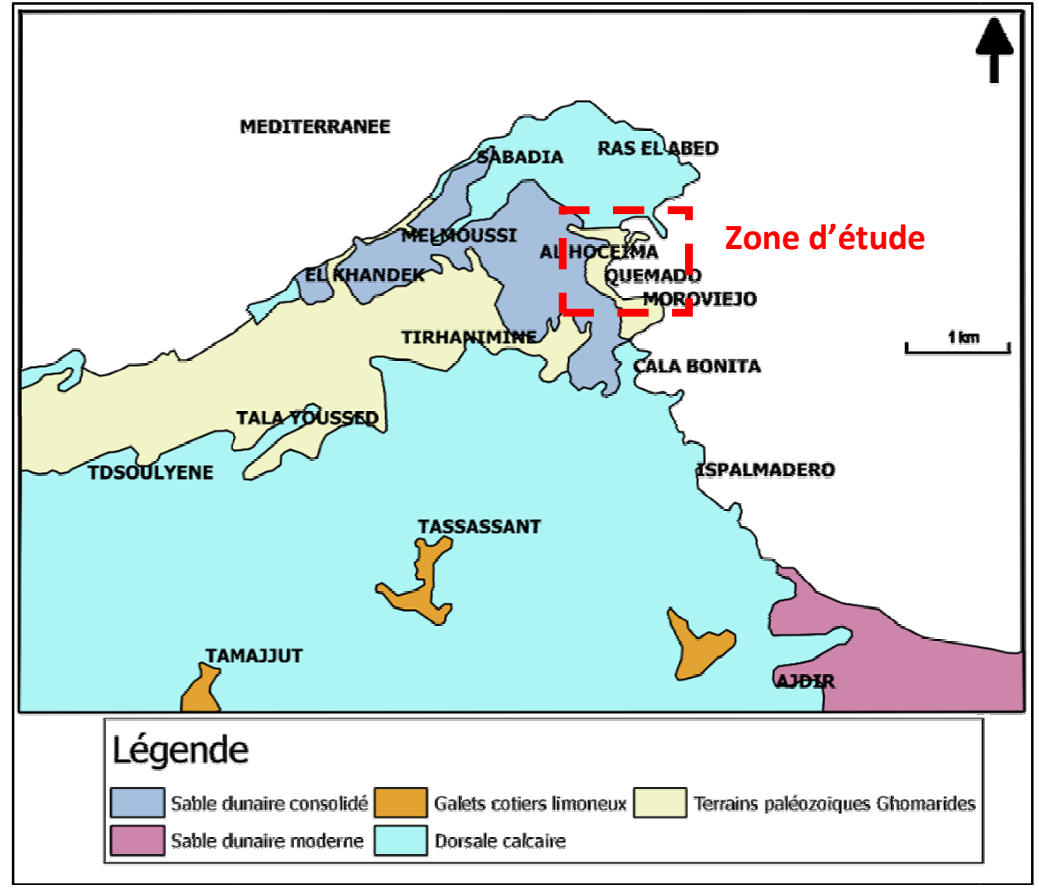

Fig.1. Cadre géologique régional d'Al Hoceima

Les affleurements carbonatés rigides (les calcaires à silex et dolomie) de la dorsale calcaire externe formant des reliefs remarquables (BOUSSEKKOUR, RAS EL ABED et JBEL ASSAGUASAGUANE) suivis des terrains paléozoïques Ghomarides dénommés localement la Klippe d'Al Hoceima. Cette Klippe s'étend d'Al Hoceima à l'oued BOUSSEKKOUR à l'Ouest avec une stratigraphie en schistes satinés et noirs (12) surmontée par des calcaires dévoniens constituant les points géomorphologiques les plus hauts (SIDI MANSOUR, TALA YOUSSEF et la zone étudiée QUEMADO).

\subsection{Cadre sismique}

La région d'Al Hoceima peut être présentée comme la plus sismogène au Maroc. Par conséquent, elle est parmi les zones actives les plus étudiées le long de la frontière de plaques diffuse Afrique-Eurasie dans la méditerranée occidentale. Les cartes de sismicité de la région montrent un important alignement sismique de direction NE-SW.

Plusieurs tremblements de terre ont pu être considérés dans l'histoire de la région grâce à l'ampleur des désastres qu'ils provoquèrent. On compte trois événements majeurs (dont les deux premiers destructeurs), en 1994, 2004 et 2016 ayant des magnitudes modérées entre 5.9 et 6.3 et des mécanismes au foyer similaires.

\subsection{Cadre géomorphologique}

L'examen du site étudié montre que le versant possède un relief à pentes irrégulière (figure 2), accentuée par l'existence de trois thalwegs larges et par l'action anthropique des aménagements existants et en cours, ce site est délimité au sommet par les constructions de la ville d'Al Hoceima tandis que le pied du versant abrite des bâtiments relevant d'un complexe touristique.

En dénivelée, le secteur possède des pentes comprises entre la plage méditerranéenne et $65 \mathrm{~m}$ d'altitude, les pentes très irrégulières dont les plus fortes dépassent $40^{\circ}$.

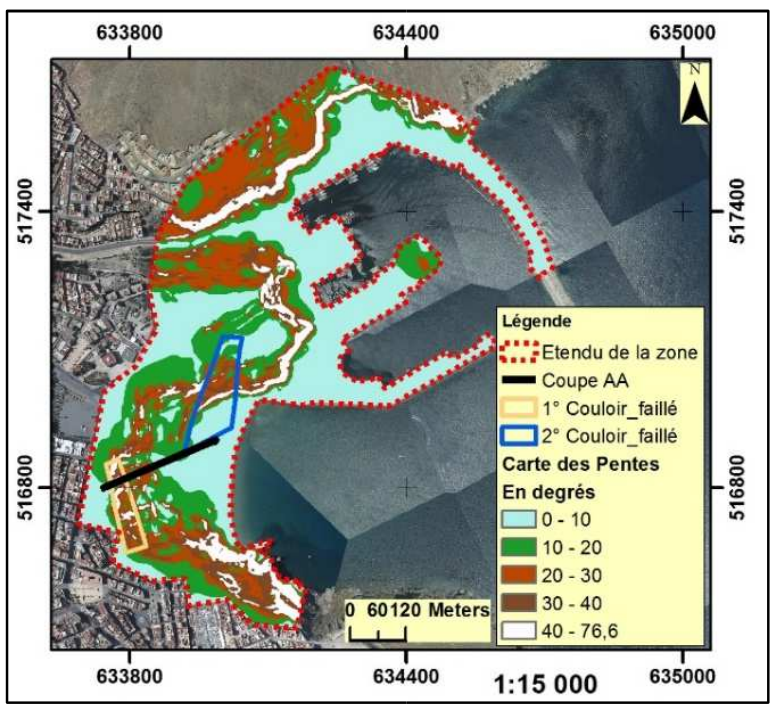

Fig. 2. Cadre morphologique du secteur d'étude

Le Versant QUEMADO présente un cas de figure de la vulnérabilité du Rif au risque «mouvements de terrain ».

Le risque étudié est favorisé par la conjonction de plusieurs paramètres qui relèvent principalement des caractéristiques structurales et topographiques du versant.

L'examen de la zone constituée du versant, reflète l'existence des pélites schisteuses, des calcaires et des dépôts de couverture meubles (figure 3). 


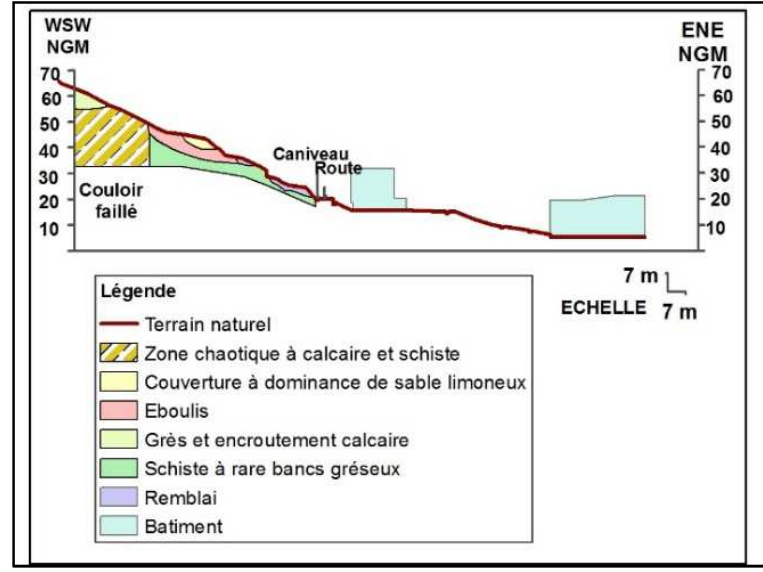

Fig. 3. Coupe géologique (AA)

\section{Problématique}

Le Versant QUEMADO est sujette au risque « mouvements de terrain », favorisé par la conjonction de plusieurs paramètres qui relèvent principalement des caractéristiques structurales et topographiques du versant.

\section{Objectifs de l'étude}

Afin de contribuer à une meilleure connaissance du risque dans l'espace d'étude, notre recherche suit une démarche transversale appuyée sur des approches permettant l'évaluation qualitative et quantitative de l'aléa. L'objectif est de caractériser les facteurs de déclenchement du risque et son évaluation à une échelle urbaine.

\section{Hypothèses de déclenchement des mouvements de terrain}

Les particularités lithologiques et géotechniques de la zone étudiée permettent de définir les hypothèses de déclenchement suivantes :

- Versant à pente raide et sol hétérogène ;

- Présence d'écoulements d'eau ;

- Charge des constructions en amont du versant ;

- Couloirs faillés et sismicité régionale.

\section{Démarche d'étude et extraction des paramètres}

\subsection{Evaluation qualitative du mouvement de terrain}

L'évaluation des risques à l'échelle urbaine est un atout $[14,15]$. Les trois grandes étapes de l'approche qualitative employée sont présentées ci-dessous.

- Cartographie de la susceptibilité au mouvement de terrain par la prise en compte des paramètres physiques ayant engendré l'aléa. L'intensité de chaque paramètre est exprimée en échelles de valeurs et pondérés par des poids [7] adaptées (Table 1) ;
- Analyse et cartographie des enjeux suivant deux catégories d'enjeux à partir des données d'occupation du sol disponibles (Table 2) ;

- Croisement des cartes de susceptibilité et d'enjeux en multipliant les valeurs de classes (Table 3).

Table 1. Paramètres de prédisposition au risque

\begin{tabular}{|c|c|c|}
\hline Paramètres & Intitulé du paramètre & Pondération \\
\hline Topographie & Pente de terrain (P) & 4 \\
\cline { 2 - 3 } & Orientation de la pente (O) & 2 \\
\hline Hydrographie & $\begin{array}{c}\text { Distance du réseau } \\
\text { hydrographique (H) }\end{array}$ & 3 \\
\hline Désordres & $\begin{array}{c}\text { Distance des désordres } \\
\text { naturels (D) }\end{array}$ & 3 \\
\hline $\begin{array}{c}\text { Géologie } \\
\text { structurale }\end{array}$ & $\begin{array}{c}\text { Susceptibilité au } \\
\text { déplacement (G) }\end{array}$ & 4 \\
\hline $\begin{array}{c}\text { Structure } \\
\text { sismique }\end{array}$ & $\begin{array}{c}\text { Distance des contacts } \\
\text { anormaux et failles (F) }\end{array}$ & 4 \\
\hline Confortement & $\begin{array}{c}\text { Distance des } \\
\text { confortements réalisés (C) }\end{array}$ & 3 \\
\hline
\end{tabular}

Table 2. Valeurs qualitatives d'endommagement attribuées

\begin{tabular}{|c|c|c|}
\hline $\begin{array}{c}\text { Carte } \\
\text { d'enjeux }\end{array}$ & Intitulé du paramètre & $\begin{array}{c}\text { Valeurs } \\
\text { attribuées }\end{array}$ \\
\hline \multirow{4}{*}{$\begin{array}{c}\text { Fon } \\
\text { Fonctionnelles } \\
\text { et } \\
\text { économiques } \\
(\mathbf{F E})\end{array}$} & $\begin{array}{c}\text { Aménagements } \\
\text { structurants }\end{array}$ & 60 \\
\cline { 2 - 3 } & Voirie & 50 \\
\cline { 2 - 3 } & Surfaces artificielles & 40 \\
\cline { 2 - 3 } & Terrain nu & 20 \\
\cline { 2 - 3 } & Espace vert & 12 \\
\cline { 2 - 3 } & Foret & 6 \\
\cline { 2 - 3 } & Habitat & 100 \\
\cline { 2 - 3 } & Equipements & 90 \\
\cline { 2 - 3 } & Tourisme & 80 \\
\hline \multirow{3}{*}{\begin{tabular}{c} 
Humaines (H) \\
\cline { 2 - 3 }
\end{tabular}} & Dépendances & 40 \\
\cline { 2 - 3 } & Forte & 90 \\
\cline { 2 - 3 } & Moyenne & 80 \\
\hline
\end{tabular}

Table 3. Croisement des cartes de susceptibilité et d'enjeux

\begin{tabular}{|c|c|c|c|c|c|}
\cline { 2 - 6 } \multicolumn{1}{c|}{} & $\begin{array}{c}\text { S1 } \\
\text { Très } \\
\text { faible }\end{array}$ & $\begin{array}{c}\text { S2 }: \\
\text { Faible }\end{array}$ & $\begin{array}{c}\text { S3: } \\
\text { Moyenne }\end{array}$ & $\begin{array}{c}\text { S4 }: \\
\text { Forte }\end{array}$ & $\begin{array}{c}\text { S5 } \\
\text { Très } \\
\text { forte }\end{array}$ \\
\hline $\begin{array}{c}\text { E1 }: \text { Très } \\
\text { faibles }\end{array}$ & $\begin{array}{c}\text { Très } \\
\text { faible }\end{array}$ & $\begin{array}{c}\text { Très } \\
\text { faible }\end{array}$ & $\begin{array}{c}\text { Très } \\
\text { faible }\end{array}$ & $\begin{array}{c}\text { Très } \\
\text { faible }\end{array}$ & $\begin{array}{c}\text { Très } \\
\text { faible }\end{array}$ \\
\hline $\begin{array}{c}\text { E2: } \\
\text { Faibles }\end{array}$ & $\begin{array}{c}\text { Très } \\
\text { faible }\end{array}$ & $\begin{array}{c}\text { Très } \\
\text { faible }\end{array}$ & $\begin{array}{c}\text { Très } \\
\text { faible }\end{array}$ & Faible & Faible \\
\hline $\begin{array}{c}\text { E3: } \\
\text { Moyens }\end{array}$ & $\begin{array}{c}\text { Très } \\
\text { faible }\end{array}$ & $\begin{array}{c}\text { Très } \\
\text { faible }\end{array}$ & Faible & Faible & Moyen \\
\hline $\begin{array}{c}\text { E4 : Forts } \\
\text { Très } \\
\text { faible }\end{array}$ & Faible & Faible & Moyen & Fort \\
\hline $\begin{array}{c}\text { E5 }: \text { Très } \\
\text { forts }\end{array}$ & $\begin{array}{c}\text { Très } \\
\text { faible }\end{array}$ & Faible & Moyen & Fort & $\begin{array}{c}\text { Très } \\
\text { fort }\end{array}$ \\
\hline
\end{tabular}

\subsection{Evaluation quantitative du mouvement de terrain}

L'approche d'évaluation quantitative du risque est fondée sur un calcul et une hiérarchisation de la valeur (monétaire ou non monétaire) [16] des éléments exposés, ainsi qu'une évaluation de la susceptibilité par la méthode de régression logistique (ou régression binomiale) [17]. 
Les cartes résultantes sont classifiées en échelle d'intensité et croisées pour extraire la carte de risque globale. La méthodologie d'évaluation du risque repose sur 2 étapes.

- Etape 1: Basée sur le calcul de la probabilité d'occurrence des mouvements pour chaque pixel de la zone d'étude :

$$
P=\frac{1}{1+e^{-z}}
$$

Où $\mathrm{z}$ représente une combinaison linéaire des paramètres prédicteurs du risque $\mathrm{X}_{1}, \mathrm{X}_{2}, \ldots, \mathrm{X}_{\mathrm{n}}$ :

$z=b+a_{1} X_{1}+a_{2} X_{2}+\cdots+a_{n} X_{n}$

Avec $\mathrm{b}$ le terme libre du modèle et les coefficients $a_{i}$ de la régression.

Les paramètres prédicteurs retenus dans le cadre de cette étude ayant un niveau élevé de représentativité sont: la pente $\left(X_{1}\right)$, l'orientation du terrain $\left(X_{2}\right)$, la distance du réseau hydrographique $\left(X_{3}\right)$, la distance des désordres naturels constatés $\left(X_{4}\right)$, la susceptibilité de déplacement des formations géologiques $\left(X_{5}\right)$, la distance des contacts anormaux et couloirs faillés $\left(X_{6}\right)$ et la distance des confortements du sol réalisés $\left(X_{7}\right)$ [18].

- Etape 2 : Basée sur l'identification et l'attribution des valeurs d'endommagement proportionnelles à la valeur monétaire des enjeux exposés (Table 4).

Table 4. Valeurs attribuées des enjeux

\begin{tabular}{|c|c|c|c|}
\hline & $\begin{array}{c}\text { Enjeu } \\
\text { structurel } \\
\text { (ES) }\end{array}$ & $\begin{array}{c}\text { Enjeu } \\
\text { fonctionne } \\
1(\mathrm{EF})\end{array}$ & $\begin{array}{l}\text { Enjeu } \\
\text { corporel } \\
\text { (EC) }\end{array}$ \\
\hline & $\begin{array}{c}\text { Valeur } \\
\text { monétaire en } \\
\text { MAD/m² } \\
\text { (Valeur } \\
\text { hiérarchisé) } \\
\end{array}$ & $\begin{array}{l}\text { Valeurs } \\
\text { attribuées }\end{array}$ & $\begin{array}{l}\text { Valeurs } \\
\text { attribuées }\end{array}$ \\
\hline $\begin{array}{l}\text { Aménagements } \\
\text { structurants ; }\end{array}$ & $600(8)$ & 60 & 50 \\
\hline Voirie ; & $400(5,3)$ & 70 & 60 \\
\hline $\begin{array}{l}\text { Surfaces } \\
\text { artificielles. }\end{array}$ & $200(2,7)$ & 50 & 45 \\
\hline - Espace vert ; & $150(2)$ & 20 & 30 \\
\hline - Foret ; & $200(2,7)$ & 40 & 25 \\
\hline - Eau ; & $100(1,3)$ & 30 & 15 \\
\hline - Sol nu. & $700(9,3)$ & 20 & 30 \\
\hline $\begin{array}{l}\text { - Plancher } \\
\text { habitable ; }\end{array}$ & $5500(73,3)$ & 100 & 100 \\
\hline $\begin{array}{l}\text { - Plancher } \\
\text { d'équipement ; }\end{array}$ & $4000(53,3)$ & 90 & 70 \\
\hline $\begin{array}{l}\text { - Plancher } \\
\text { touristique. }\end{array}$ & 7500 (100) & 80 & 80 \\
\hline
\end{tabular}

\section{Résultats}

\subsection{Approche qualitative}

La carte de risque qualitatif (figure 4) est retrouvée par croisement des cartes classifiées de susceptibilité et enjeux qualitatives (Tableau 3).

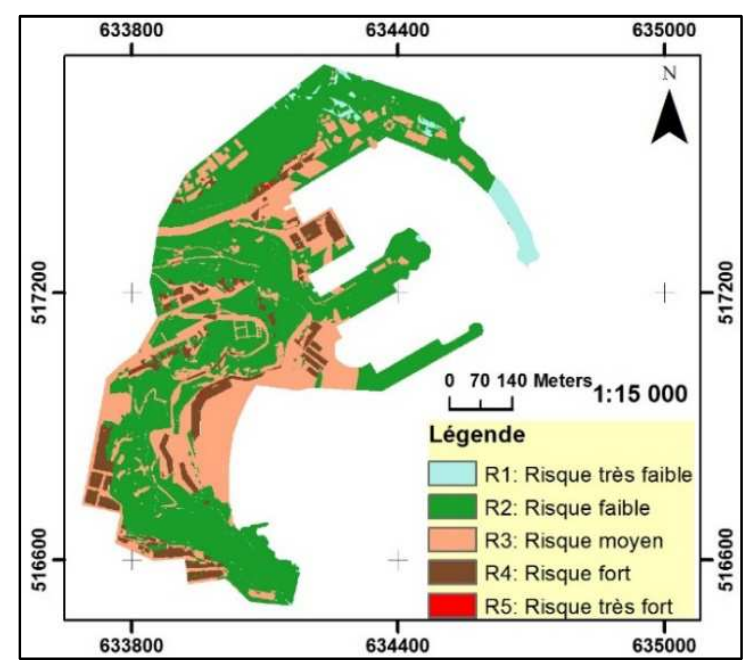

Fig. 4. Carte du risque qualitatif des mouvements de terrain

\subsection{Approche quantitative}

L'application du de la régression binomiale a permis d'extraire la carte de susceptibilité quantitative aux mouvements de terrain croisée à la carte des enjeux de la zone. La carte de risque quantitatif retrouvée est présentée par la figure 5 .

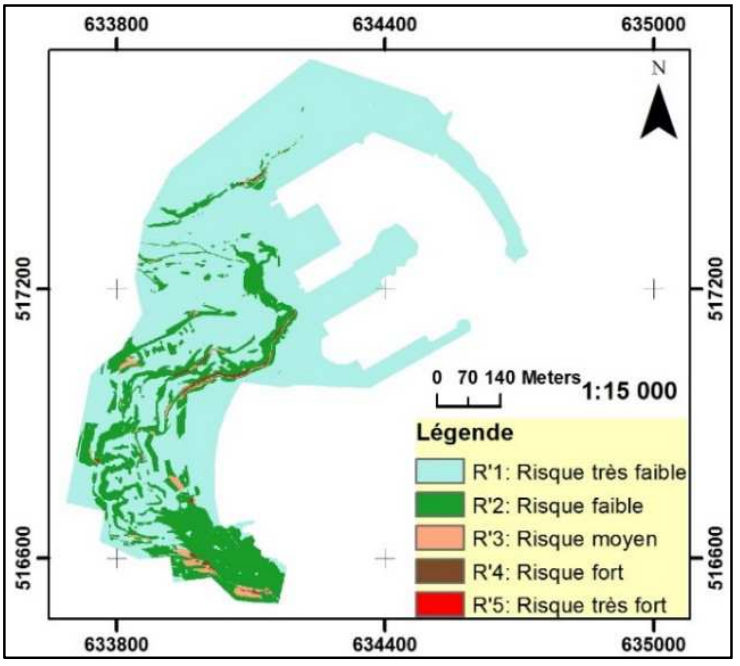

Fig. 5. Carte du risque quantitatif des mouvements de terrain

\section{Discussion}

La zone d'étude est sujette au risque de mouvement de terrain vue son contexte géologique et morphologique. A cet effet, l'étude a nécessité l'introduction des paramètres prédicteurs du risque.

L'affectation des poids à chaque paramètre de prédisposition est une étape décisive dans la perception de l'aléa et exige impérativement une connaissance des particularités de la zone et le jugement de l'expert.

La comparaison des pondérations intégrées dans le modèle qualitatif aux coefficients calculés par la régression logistique remet en lumière le rôle essentiel que jouent les formations géologiques et la pente 
topographique dans la répartition des mouvements de terrain à région d'Al Hoceima [21] (Table 5).

Table 5. Coefficients de pondération des deux approches

\begin{tabular}{|c|c|c|}
\hline $\begin{array}{c}\text { Paramètres de } \\
\text { prédispositions }\end{array}$ & $\begin{array}{c}\text { Coefficients de } \\
\text { pondération } \\
\text { retrouvés par } \\
\text { régression }\end{array}$ & $\begin{array}{c}\text { Coefficients de } \\
\text { pondération } \\
\text { introduits } \\
\text { qualitativement }\end{array}$ \\
\hline Pente & 0,088 & 4,0 \\
\hline Orientation de la pente & 0,000 & 2,0 \\
\hline $\begin{array}{c}\text { Distance du réseau } \\
\text { hydrographique }\end{array}$ & 0,005 & 3,0 \\
\hline $\begin{array}{c}\text { Distance des désordres } \\
\text { naturels }\end{array}$ & 0,011 & 3,0 \\
\hline $\begin{array}{c}\text { Susceptibilité } \\
\text { géologique }\end{array}$ & 0,255 & 4,0 \\
\hline $\begin{array}{c}\text { Distance des contacts } \\
\text { anormaux et failles }\end{array}$ & 0,004 & 4,0 \\
\hline $\begin{array}{c}\text { Distance des } \\
\text { confortements }\end{array}$ & $-0,002$ & 3,0 \\
\hline
\end{tabular}

Les résultats acquis par application des démarches présentées au paragraphe 6 ont permis d'établir deux cartographies ayant des proportions du risque différentes.

A cette analyse, une mission d'investigation terrain a été exécutée pour évaluer la pertinence des résultats. L'analyse nous ramène à subdiviser celle-ci en 2 catégories (figure 6) :

- Catégorie 1: L'emprise des talwegs situés en amont des projets touristiques que connait des signes d'instabilité plus ou moins prononcés (désordres, couloirs faillés, caractéristiques mécaniques faibles du sol) avec des pentes raides et moyennes ;

- Catégorie 2 : La partie reliant les talwegs à la limite du versant qui a une apparence relativement stable avec des pentes faibles et un aménagement contribuant à la stabilisation du terrain.

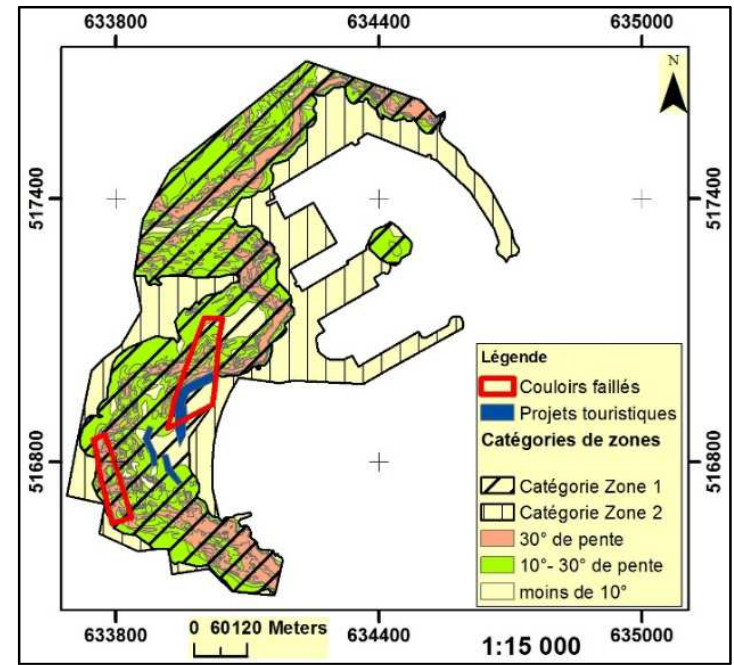

Fig. 6. Constatations et catégories spatiales de risque

La confrontation de cette cartographie aux cartes de susceptibilité précédentes montre que l'approche quantitative est plus proche en résultats, offre un zonage plus fin et des poids objectifs attribués à chaque paramètre de prédisposition.

\section{Conclusion}

Comme il a été décrit dans des travaux antérieurs (4), la répartition des mouvements de terrain se concentre à proximité des falaises ou le long des versants accidentés des principaux cours d'eau. L'examen du secteur d'étude montre que cette donnée se manifeste clairement et coïncide également avec une proximité des principales failles de la région.

La pertinence des modèles qualitatifs et quantitatifs d'évaluation du risque dépend du type, de la densité et de la qualité des données existantes.

L'approche quantitative est plus objective (calcul des poids de façon probabiliste et qualification des enjeux à la base des valeurs réel des enjeux exposés) et fournie des résultats qui se confirment par les investigations et les relevés terrain. Cette cartographie produite par des données de base au $2000^{\text {ème }}$ en exploitant les fonctionnalités du système d'informations géographiques (SIG) porte un complément reproductible à d'autres zones à risques.

\section{Références}

1. Millies-Lacroix, Les glissements de terrain, présentation d'une carte prévisionnelle des mouvements de terrain dans le Rif. Mine et Géologie, 27, pp. 45-54 (1968)

2. B. El Fellah, Eboulement rocheux dans le Paléozoique du Rif: Présentation d'un cas sur la route Oued Lao-Jebha. Lisboa, Portugal: 7ème Congré Intern. de l'AIGI, pp. 3927- 3931 (1994)

3. B. El Fellah, O. Azzouz, L. Assebriy, Exemple de glissement de terrain littoral sur la cote méditerranéenne des Bokoya entre Torrès et Badis, Rif, Maroc. ORSTOM, Réseau érosion, 16 (1996)

4. K. Margua, Essai de cartographie des risques naturels: Application à l'aménagement de la région d'Al Hoceima. Thèse universitaire, Univ. FrancheComté, 196 p (1994)

5. A. Talhaoui, M. Aberkan, A. Iben Brahim, A. El Mouraouah, N. Jabour, Contribution à l'étude des risques naturels. Cas de la région d'Al Hoceima. Oujda : Colloque des Bassins Sédimentaires Marocains (1999)

6. Y. Moriwacki, I.M. Idrissi, T.L. Moses, R.S. Ladd, A re-evaluation of the " $L$ " Strret Slide in Anchorage during the 1964 Alaska earthquake . Rio de Janeiro : Comtes rendues XII ICSMFE, 3, pp. 1583-1586 (1985)

7. M. Levasseur, Contribution des systèmes d'information géographique à l'analyse quantitative de l'aléa "glissement de terrain" - Exemple d'application au secteur de Ville de la Baie, Québec. Québec : Université de Québec (2003)

8. F. Léone, Concept de vulnérabilité appliqué à l'évaluation des risques générés par les phénomènes 
de mouvements de terrain. Grenoble: Rapport de thèse, Université Joseph Fourier (1996)

9. J.P. Malet, H. Thiery, O. Maquaire, A. Puissant, Analyse spatiale, évaluation et cartographique du risque "glissement de terrain". Revue Internationale de Géomatique, 10, pp. 1-10 (2006)

10. M.M. Blumenthal, Esbozo geologico del Rif en la region de Bokoya. Bol. Inst. Geol. Min. Espana, Madrid., 44, pp. 199-352 (1937)

11. J. Andrieux, La structure du Rif central, étude des relations entre la tectonique de compression et les nappes de glissements dans un tronçon de la chaine alpine . Notes et Mém. Serv. Géol. Maroc, 235, 155 $\mathrm{p}(1971)$

12. T. Mouriet, Etude géologique et structurale $d u$ massif des Bokoya . Trav. Lab. Géol. de l'Afrique . Univ. Paris Sud, (1982).

13. O. Azzouz, La lithostratigraphie et tectonique des terrains paléozoiques Ghomarides du massif des Bokoya (Rif interne, Maroc). Thèse $3^{\circ}$ cycle .Rabat, 208 p (1992)

14. M. Michael-Leiba, F. Baynes, G. Scott, K. Granger, Regional landslide risk to the Cairns community. Natural Hazards, 30, Issue 2, pp. 233-249 (2003)

15. G. Grünthal, A.H. Thieken, J. Schwarz, K. Radtke, A. Smolka, B. Merz, Comparative risk assessment for the city of Cologne, Germany - storms, floods, earthquakes. Natural Hazards, 38, pp. 21-44 (2006)

16. T. Glade, Vulnerability assessment in landslide risk analysis, 134, pp. 121-138 (2003)

17. Y. Thiery, J.P. Malet, S. Sterlacchini, A. Puissant, O. Mequaire, Landslide susceptibility assessement by bivariate mehods at large scales : Application to a complex mountainous environment. Geomorphology, 92, pp. 38-59 (2007)

18. M.C. Margarint, A. Grozavu, C.V. Patriche, A-M.I. Tomasciuc, R. Urdea, I. Ungurianu, Evaluation des risques de glissements de terrain par la méthode de régression logistique : Application à deux zones basses de Roumanie . Dynamiques environnementales. LGPA-EDITIONS, 28 (2011)

19. M. Mejia Navarro, E.E. Wohl, S.D. Oaks, Geological Hazards, vulnerability, and risk assessment using GIS : Model for Glenwood Springs. Geomorphology, 10, pp. 331-354 (1994)

20. S. Aronoff,Geographic Information Systems:A Management Perspective.WDLPublications, 294 p (1989)

21. A. Talhaoui, M. Aberkan, Iben Brahim, A. El Mouraouah, Risques géologiques et activité sismique dans la région d'Al Hoceima (Maroc) : Approche de la quantification des facteurs responsables $d u$ déclenchement des instabilités de terrain. Pangea, 43, pp. 3-18 (2005) 